\title{
EVALUACIÓN DE LAS VÍAS AÉREAS SUPERIORES A TRAVÉS DE TRAZADOS CEFALOMÉTRICOS
}

\section{UPPER AIRWAYS EVALUATION THROUGH CEPHALOMETRIC TRACINGS}

\author{
Quevedo-Piña Maira* \\ Hernández-Andara Adalsa** \\ Zambrano Elba"** \\ Domingos Vanda ${ }^{* * * *}$
}

\section{RESUMEN}

La cefalometría es un elemento auxiliar de diagnóstico utilizada frecuentemente en el estudio del crecimiento y desarrollo cráneofacial; y en la planificación de terapéuticas de ortodoncia y ortopedia funcional de los maxilares. Además se considera una herramienta importante para evaluar el espacio aéreo nasofaríngeo, ya que, gracias a la observación de los relieves óseos y perfil blando del paciente, se obtiene una información estática y bidimensional sobre el calibre de las vías aéreas superiores. Así, el objetivo del presente artículo es analizar una selección de trazados cefalómetricos orientados a evaluar este reparo anatómico. En tal sentido se realizó una búsqueda por diferentes bases de datos, tales como Pubmed, Medline, Scielo y Google Scholar, en las que se obtuvo información sobre diferentes cefalometrías utilizadas para lograr el propósito de la presente investigación. Los resultados obtenidos en la revisión concluyen que el criterio de selección dependerá fundamentalmente de la información que el clínico desea obtener para cubrir los requerimientos del paciente.

Descriptores: Cefalometría · Ortodoncia

\section{ABSTRACT}

Cephalometrics is a tool for the study of craniofacial growth and development; it is considered an important technique to evaluate nasopharyngeal air space, and due to the observation of bony prominences and soft profile of the patient, we can get a two-dimensional and static information about the caliber of the upper airway. Thus, this article aims to present a selection of cephalometric tracings to evaluate upper airway, for which a search for different databases, such as PubMed, Medline, Scielo and Google scholar, among others, in the information on different paths used to achieve the purpose of the investigation was obtained. In conclusion, it was established that the selection criteria depend fundamentally on the information that the clinician want to get to meet the requirements of the patient.

Descriptors: Cephalometry · Orthodontics

\footnotetext{
* Facultad de Odontología. Universidad de Carabobo. Venezuela.

** Clínica Félix Boada. Venezuela.

*** Centro Odontológico Zambrano. Venezuela.

**** Universidade São Leopoldo Mandic. Brasil.
} 


\section{NTRODUCC I ÓN}

La cefalometría, en sus inicios, se desarrolló como una técnica a ser usada en antropología, para cuantificar la forma y dimensiones del cráneo. Fue Broadbent quien la introduce en el campo de la odontología y, a partir de ese momento, ha sido empleada como una importante herramienta auxiliar en el diagnóstico clínico y la investigación, sobre todo cuando se requiere un análisis para la interpretación de la calidad y dirección del crecimiento y desarrollo craneofacial. La radiografía cefálica lateral se indica con mayor frecuencia, ya que permite la visualización, no solo de la morfología esquelética, dental y de tejidos blandos, sino también de sus relaciones espaciales, permitiendo la interpretación de la cantidad y dirección de crecimiento, y de la presencia o no de anomalías craneofaciales, contribuyendo en la planificación y evaluación del tratamiento. ${ }^{1}$

Con la imagen de la radiografía cefálica lateral es posible evaluar, el espacio aéreo nasofaringeo ${ }^{2}$, ya que gracias a la observación de los relieves óseos y perfil blando del paciente, se obtiene una información estática y bidimensional sobre las condiciones de las vías aéreas superiores (VAS), que permiten precisar e identificar, el lugar de la obstrucción respiratoria (si la hubiese) lo que contribuye, en algunos casos, a la toma de decisiones de terapéuticas ${ }^{3}$. También ha sido ampliamente utilizada para evaluar las estructuras óseas y blandas en pacientes con apnea obstructiva del sueño; dentro de las que se incluyen: el estrechamiento del espacio aéreo posterior, paladar blando hipertrófico y posicionamiento inferior del hioides, retroposición mandibular y ángulo nasion-sella-basion (ángulo de la flexión craneana) pequeño ${ }^{4}$.

La radiografía cefálica lateral es el recurso auxiliar de diagnóstico ideal, porque además de permitir la realización de trazados que nos cuantifiquen la presencia o no de desarmonías, se obtiene a través de un cefalostato, el cual permite generar imágenes estandarizadas que pueden ser reproducidas en la misma posición de referencia, lo que permite evaluar, medir y comparar los cambios por tratamiento 0 bien por crecimiento ${ }^{5}$

Las vías aéreas superiores, conocidas también como espacio nasofaríngeo, constituyen una estructura compleja y multifuncional, que regula diferentes funciones del organismo contradictorias entre sí, ya que coordinan funciones respiratorias y de ventilación, así como funciones gástricas y de fonación. Forman parte de ellas: las fosas nasales, faringe y laringe, estructuras estas que juegan un papel crucial en la vida del ser humano. Las fosas nasales humedecen, filtran y calientan el aire inspirado; la faringe permite el paso de alimentos sólidos y líquidos además del aire ${ }^{6}$; por su parte la laringe, considerada el límite entre las VAS y las vías aéreas inferiores, juega un papel importante en la fonación además de tener una función de protección de a las vías respiratorias inferiores, de manera que cuando una partícula, por accidente, pasa hacia la tráquea, ésta es expulsada a través del reflejo tusígeno ${ }^{7}$.

Durante la respiración nasal es fundamental que se produzca el cierre bucal, lo que puede ocurrir normalmente por el sellamiento labial, o bien por el contacto del dorso y la parte posterior de la lengua con el paladar duro y blando respectivamente. La esfera maxilo-faringo-bucal constituye lo que los neurofisiólogos denominan el efector común, porque órganos diversos inervados por el mismo tronco común, ejercen diversas funciones todas discretas individualmente, pero que adquieren una alta jerarquía cuando se integran unas con otras, de tal manera que la especialización de los órganos faringo-bucales, está más relacionada por las funciones que los unen que por su fisiología ${ }^{8}$. Es por ello que, a pesar que la lengua no forma parte del sistema respiratorio, tiene una amplia participación en la respiración. La lengua es una estructura muscular compleja, que posee tres puntos de inserción; uno óseo fijo en la mandíbula a través del músculo geniogloso; el segundo móvil en el hueso hioides dado por músculo hiogloso y un tercero muscular que corresponde a los músculos intrínsecos.

La posición de la lengua es de suprema
QUEVEDO-PIÑA M HERNÁNDEZ-

ANDARA A

ZAMBRANO E

VANDA D

EVALUACIÓN DE

LAS VÍAS AÉREAS

SUPERIORES

A TRAVÉS DE

TRAZADOS

CEFALOMÉTRICOS

277
REV. ODONTOL.

UNIV. CID, SÃo

PAULO

2017; 29(3):

$276-88$, SET - DEZ 
QUEVEDO-PIÑA $M$

HERNÁNDEZ-

ANDARA A

ZAMBRANO E

VANDA D

EVALUACIÓN DE LAS VIIAS AÉREAS

SUPERIORES

A TRAVÉS DE

TRAZADOS

CEFALOMÉTRICOS

\section{8}

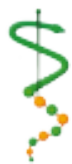

REV. ODONTOL, UNIV. CID. SÃO

PAULO

2017; 29(3): $276-88$, SET - DEZ importancia para el equilibrio de la musculatura bucofacial; así lo reflejan diversos estudios en los que se han detectado variaciones en la región orofaríngea, en pacientes con diferentes patrones esqueletales, específicamente en el segmento a nivel de la base de la lengua hasta la pared posterior faríngea. En una investigación desarrollada por Han et al. ${ }^{9}$ (2014), se concluyó que los adolescentes con maloclusión Clase II, tenían la relación lengua-pared posterior de la faringe, significativamente menor cuando fueron comparados con los clasificados como clase I; de allí la importancia de incluir la posición de la lengua en el estudio de las vías aéreas superiores.

En pacientes con apnea obstructiva del sueño, los sitios de obstrucción y el estrechamiento de las VAS difieren considerablemente; pero las regiones mayormente afectadas son la retropalatal y la región posterior a la base de la lengua. En este sentido, el análisis cefalométrico constituye una herramienta muy valiosa para el diagnóstico y planificación del tratamiento de estos pacientes, permitiendo estudiar la morfología dentofacial, para evaluar la existencia de retrognatia o micrognatia, y analizar las condiciones del ángulo ANB, la inclinación del plano mandibular, el posicionamiento del hueso hioides, las dimensiones del paladar blando y el tamaño de la lengua ${ }^{10}$.

En condiciones de reposo, el respirar por la nariz requiere más esfuerzo que hacerlo por la boca, dado que los conductos nasales, cuya función es calentar, humedecer y filtrar el aire inspirado, representan una resistencia al flujo respiratorio. Si la nariz está obstruida parcialmente, aumenta el trabajo para inspirar y al llegar a un nivel determinado de resistencia al flujo respiratorio, el individuo adopta la respiración bucal parcial, de tal manera que los cambios en las dimensiones del tracto respiratorio por constricción u obstrucción, pueden disminuir el flujo del aire y los músculos respiratorios pueden incrementar su trabajo; producto de esto, se implementa el flujo del aire por la cavidad bucal, lo que trae como consecuencia una alteración de la función de los músculos intrínsecos y extrínsecos de las VAS, que modifica la posición de la mandíbula y la lengua e influye en la morfología y crecimiento esquelético facial ${ }^{11}$.

La cefalometría es de gran utilidad para monitorear estos cambios, particularmente en los pacientes con ortodoncia. Sin embargo, existen algunas limitaciones porque solo puede observarse la progresión lineal, vertical y anteroposterior del cambio facial, estando limitada su capacidad para describir características tridimensionales. Para evaluar en los tres planos del espacio (frontal, coronal y axial), deben utilizarse otros métodos de estudio como son la tomografía Cone Beam (CBCT) y la resonancia magnética (RM), que permiten mediciones precisas en tres dimensiones (3D), de las diversas áreas de la anatomía de las vías respiratorias, a través de imágenes con reconstrucciones volumétricas.

La resonancia magnética, a pesar de no utilizar radiación ionizante, requiere un tiempo operativo significativamente mayor, que se traduce en disminución de la calidad de la imagen de las vías respiratorias, debido al movimiento del paciente condicionado por la presencia de artefactos. Por tal razón, de estos métodos de estudio por imagen, el CBCT ha conducido a una mejor comprensión de la anatomía y fisiología de las vías respiratorias superiores $^{12}$; sin embargo, existen investigaciones contradictorias en cuanto a su utilización como examen de rutina, debido al mayor volumen de tejido irradiado, baja resolución de tejidos faciales y su alto costo $^{13}$. Otro factor a considerar es la precaución que hay que tener cuando se trata de pacientes jóvenes y en ese aspecto, el principio ALARA (la radiación tan baja como sea razonablemente posible), debe prevalecer cuando se trata de seleccionar un método con radiación ionizante para diagnosticar un paciente ${ }^{14,15}$.

Existen investigaciones que validan a la cefalometría para el estudio de las VAS ${ }^{16-}$ 18, siendo uno de sus pioneros Solow ${ }^{19}$ (1966), quien propuso una serie de puntos y líneas, algunos de los cuales están aún vigentes. Desde esa fecha hasta la actualidad, se han descrito diferentes puntos cefalométricos para evaluar el espacio aéreo superior o las VAS. Otros autores los uti- 
lizan, para relacionar la postura cráneo-cérvico-mandibular con los diámetros antero posteriores de la vía aérea faríngea y sostienen que hay mecanismos de adaptación fisiológica, que permiten mantener permeable esta región ${ }^{20}$.

Cárdenas et al. afirman que la posición craneal juega un papel fundamental dentro del equilibrio cráneo-cérvico-mandibular, lo que indica que sus componentes tienen el potencial de influirse recíprocamente. La localización de las estructuras de este sistema se relacionan con características y funciones anatómicas específicas como: la posición lingual, la postura cervi- cal y mandibular ${ }^{21}$. En 1977 Solow y Kreiborg ${ }^{22}$ propusieron una hipótesis para explicar los diferentes conjuntos de asociaciones. Las vías respiratorias nasofaríngeas obstruidas y una resistencia respiratoria nasal grande, determinada rinomanométricamente fueron observadas, en relación con un gran ángulo craneocervical y con pequeñas dimensiones mandibulares, retrognatismo mandibular, gran rotación mandibular y retro-inclinación de los incisivos superiores. Las correlaciones observadas estuvieron de acuerdo con el patrón predicho de asociación entre morfología craneofacial, angulación

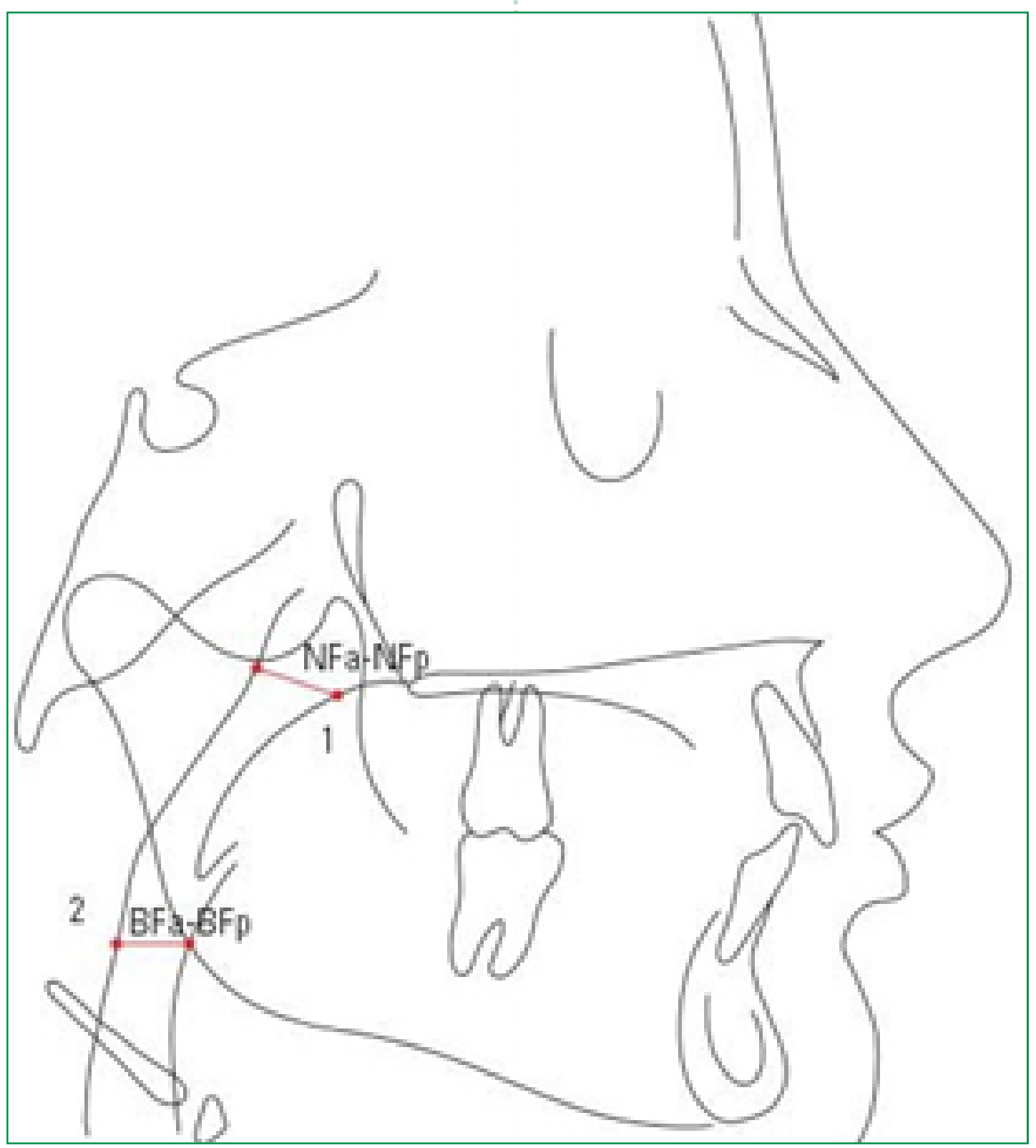

QUEVEDO-PIÑA M HERNÁNDEZ-

ANDARA A

ZAMBRANO E

VANDA D

EVALUACIÓN DE

Las VÍAS aÉREAS

SUPERIORES

A TRAVÉS DE

TRAZADOS

CEFALOMÉTRICOS 
QUEVEDO-PIÑA M

HERNÁNDEZ-

ANDARA A

ZAMBRANO E

VANDA D

EVALUACIÓN DE

LAS VIIAS AÉREAS:

SUPERIORES

A TRAVÉS DE

TRAZADOS

CEFALOMÉTRICOS

Cuadro 1. Puntos de referencia utilizados en el análisis cefalométrico

\begin{tabular}{|l|l|}
\hline Ba & Basión. Punto más anteroinferior del foramen magnum \\
\hline $\mathrm{Na}$ & Nasión. Punto más anterior de la sutura fronto nasal \\
\hline $\mathrm{Pm}$ & Pterigomaxilar o espina nasal posterior. Punto más posterior del maxilar \\
\hline $\mathrm{S}$ & Centro de la imagen de la silla turca del hueso esfenoides \\
\hline $\begin{array}{l}\text { Punto } \\
\text { A }\end{array}$ & $\begin{array}{l}\text { Parte más profundo de la concavidad que va desde la espina nasal anterior hasta el } \\
\text { reborde alveolar }\end{array}$ \\
\hline $\begin{array}{l}\text { Punto } \\
\text { B }\end{array}$ & Punto más profundo de la concavidad que va desde el reborde alveolar al mentón \\
\hline ANS & Espina nasal anterior. Punto más anterior del maxilar \\
\hline
\end{tabular}

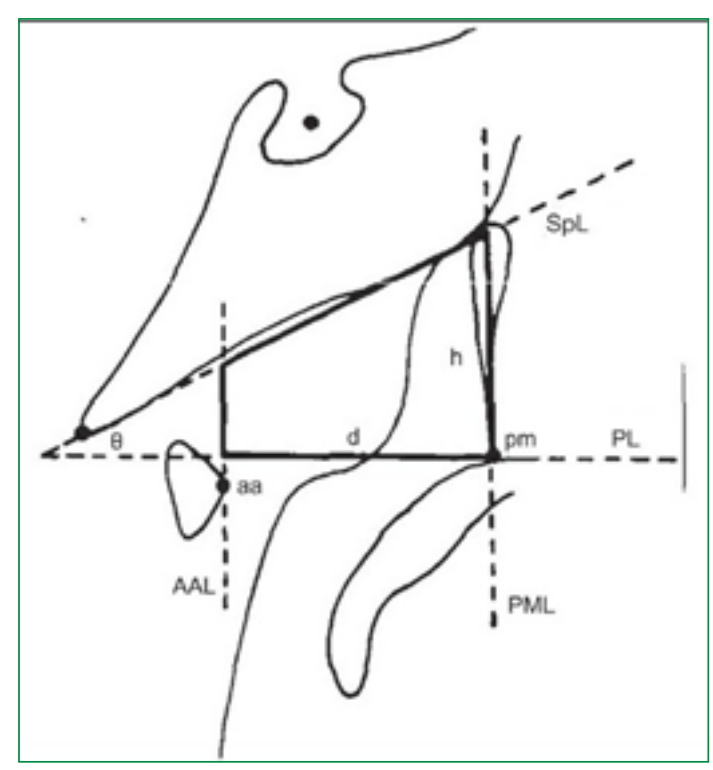

Figura 3. Análisis trapezoidal de Handleman y Osborne

craneocervical y resistencia a las vías aéreas ${ }^{22}$.

Por lo antes mencionado, se puede inferir que la cefalometría tiene un papel preponderante en el estudio de las VAS y por tal motivo, el objetivo de la presente investigación, es revisar los diferentes trazados cefalométricos usados para su evaluación, describir los usos y aplicaciones de cada cefalograma, con la finalidad de proveer herramientas que le permitan al clínico realizar una selección precisa, basada en los requerimientos diagnósticos del paciente.

\section{CEFALOGRAMA DE MCNAMARA}

Este análisis publicado en 1984, se basa principalmente en medidas lineales y no angulares, en las cuales se relacionan básicamente el maxilar con la base craneal, el incisivo superior con el maxilar, incisivo inferior con mandíbula e incorpora el análisis del tracto respiratorio a nivel de la nasofaringe y orofaringe $\mathrm{e}^{23,24}$. Para el autor, el espacio nasofaríngeo denominado NFa-NFp, es una medida lineal trazada desde un punto situado en el contorno del paladar blando, hasta el más cercano de la pared posterior de la faringe. El valor promedio para adultos, tanto hombres como mujeres, es de $17,4 \mathrm{~mm}$; en niños de 9 y 11 años se ubica en 11 y 14 mm respectivamente. Si esta medida disminuye, se podrá sospechar de una posible obstrucción por vegetaciones adenoideas.

Por su parte, el espacio orofaringeo BFa-BFp, se mide desde la intersección del borde posterior de la lengua, con el borde inferior de la mandíbula, hasta el punto más cercano en la pared posterior de la faringe, a nivel de la ubicación de las amígdalas faríngeas. El valor promedio para niños de 9 años es $11 \mathrm{~mm}$, de 11 años $12 \mathrm{~mm}$ y en el adulto de 12 a $13 \mathrm{~mm}^{25,26}$ (Fig. 1). Al contrario de lo que ocurre en la nasofaringe, no suele presentarse una obstrucción de la vía faríngea inferior por la posición de la lengua contra la faringe, pero si la distancia es mayor a $15 \mathrm{~mm}$, indica una posición adelantada de la lengua, bien sea por posición habitual o por un aumento del tamaño de las amígdalas faríngeas ${ }^{27}$.

Según estos autores, el patrón de crecimiento facial está relacionado con el patrón de crecimiento nasofaríngeo y para tal fin, utilizaron una evaluación que incluye varios puntos anatómicos: Basión (Ba), Nasión ( $\mathrm{Na}$ ), Pterigomaxilar (Pm) o espina nasal posterior, Silla (S), punto A, punto $B$, espina nasal anterior (ANS) y unas líneas de referencia, de las cuales se derivaron 6 ángulos faciales al intersectar las líneas: plano mandibular/plano silla Nasión (ML/ 
NSL), plano mandibular/plano palatal (ML/PL), plano silla Nasión/ plano palatal (NSL/PL), Nasión-Silla-Basión (Na-S-Ba), Nasión-Silla-punto A (Na-S-A) y Nasión-Silla-punto B (Na-S-B) ${ }^{28}$.

Las líneas a ser consideradas son: línea Palatal (PL), línea Atlas (AAL), línea tangente perpendicular a la proyección de la línea palatal (PL) que pasa por el punto aa (pto anterior del cuerpo del atlas); línea pterigomaxilar $(\mathrm{PML})$, perpendicular al plano palatino que cruza a nivel de la fisura pterigomaxilar; línea esfenoides $(\mathrm{SPL})$, tangente que pasa por el borde inferior del hueso esfenoides culminando en basión. Estas líneas anteriormente mencionadas $\mathrm{PL}, \mathrm{SPL}, \mathrm{AAL}$ y $\mathrm{PML}$, representan los 4 lados de un trapecio que define el área nasofaríngea ( $\mathrm{Np}$ ), que a su vez está subdividida en dos áreas: nasofaríngea (AA) y faríngea adenoidea (Ad). ( Fig. 2) ${ }^{28}$.

El área nasofaríngea ( $\mathrm{Np}$ ) se deriva matemáticamente de la fórmula $\mathrm{Np}=\mathrm{d}(\mathrm{h}-\mathrm{d}$ tan 0/ 2), donde d es igual a la profundidad, a la distancia entre Pm y el punto de intersección de las líneas PL y AAL, medida en milímetros ( $\mathrm{mm}$ ); ésta significa la profundidad antero-posterior de la nasofaringe y $\mathrm{h}$ la altura, que es la distancia entre pm y el punto de intersección de las líneas de PML y SPL medido en $\mathrm{mm}$. La altura anterior del espacio óseo nasofaríngeo o ángulo Theta, está formado por la intersección de la línea esfenoides y la línea palatina. El área aérea (AA) es medida utilizando un planímetro polar, instrumento que actualmente está en desuso y permitía hallar el área de una superficie irregular. El área adenoidea Ad se calculaba restando el área nasofaríngea del área aérea ${ }^{29}$.

Grewal y Godhane utilizan la fórmula de Handelman y Osborne para el cálculo de del espacio nasofaríngeo $(\mathrm{Np})$ y el programa Autocad para el cálculo del área adenoidea (Ad), mediante los siguientes puntos de referencia cefalométricos: $\mathrm{Ba}$, Ptm, y el centro de la silla turca (punto $\mathrm{S}$ ). So, el punto medio de la línea que une Silla turca y basión; la intersección de la línea de Ptm-Ba y la pared posterior nasofaríngea denominado Ad 1 y la intersección de la pared posterior nasofaríngea y la línea de Ptm-So es Ad 2.
Una vez obtenidas las referencias se derivan las medidas lineales: Ptm-Ad1, distancia lineal desde el punto Ptm al punto Ad1 en mm; Ptm-Ad2, distancia lineal desde el punto Ptm hasta el punto Ad2, en $\mathrm{mm}$ y con estos valores, ya se tienen las mediciones de área $\mathrm{Np}$ o zona nasofaríngea, es decir, las dimensiones verticales y antero-posterior medidas en $\mathrm{mm} 2$. Es importante considerar en los casos de pacientes respiradores bucales, que un espacio nasofaríngeo óseo constante ante el crecimiento desbalanceado de las adenoides, puede dar lugar a la obstrucción de este espacio, de allí la importancia de estas medidas ${ }^{30}$.

Por otra parte, la profundidad de la nasofaringe, que se mide desde PNS hasta Basión, aumenta en 9\% aproximadamente durante el desarrollo del paciente. La mayor parte del crecimiento nasofaríngeo, se produce en una dirección vertical, causado por el crecimiento hacia abajo tanto del paladar como de la sincondrosis esfenooccipital. En condiciones normales, la nasofaringe aumenta de tamaño en la niñez, a diferencia del tejido linfoide de la pared posterior de la nasofaringe que tiende a disminuir durante y después de la pubertad ${ }^{31}$.

La principal ventaja de este trazado, es la medición en volumen del área nasofaríngea, aunque originalmente lo descrito por Handelman y Osborne ameritaba una fórmula matemática para su cálculo y la utilización de un planímetro. En la actualidad, con la aplicación de programas informáticos como Autocad, puede obtenerse la medida del área de superficie, lo que permite al clínico evaluar la obstrucción en volumen e implica mayor rapidez en el cálculo. Otra ventaja es la existencia de valores de referencia sobre las medidas lineales y de superficie, los cuales fueron obtenidos con los cefalogramas de pacientes con función respiratoria normal. Este trazado no contempla la evaluación de la orofaringe.

CEFALOGRAMA DE LYBERG, KROGSTAD, DJUPESLAND CON MODIFICACIONES DE SAMMAN, MOHAMMADI Y XIA

Este método se basa en lo definido por Lyberg, los aportes de Tangugsorn et al. ${ }^{30}$
QUEVEDO-PIÑA M HERNÁNDEZ-

ANDARA A

ZAMBRANO E

VANDA D

EVALUACIÓN DE

LAS VÍAS AÉREAS

SUPERIORES

a tRAVÉS DE

TRAZADOS

CEFALOMÉTRICOS

281

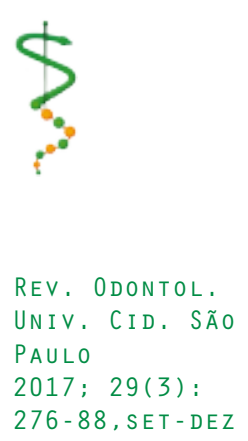




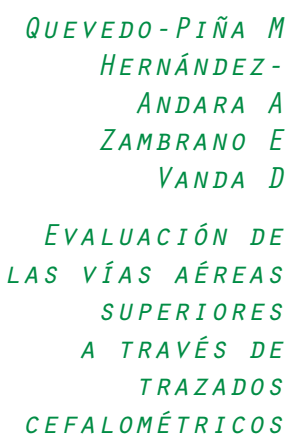

282

Cuadro 2. Definición de las medidas lineales en mm y de ángulos medidos en grados.

\begin{tabular}{|c|c|}
\hline Or & Orbitale .Punto más inferior del borde inferior de la órbita. \\
\hline Ptm & $\begin{array}{l}\text { Pterigomaxilar. Punto en el cruce de la fosa pterigo-maxilar y la espina nasal poste- } \\
\text { rior }\end{array}$ \\
\hline Po & $\begin{array}{l}\text { Porion. Punto medio de la línea que conecta al punto superior del conducto auditivo } \\
\text { externo en ambos lados }\end{array}$ \\
\hline $\mathrm{T}$ & Punta de la lengua \\
\hline U & Punta de la úvula. \\
\hline ANS & Espina nasal anterior. Situada en el plano medio sagital. \\
\hline PNS & $\begin{array}{l}\text { Espina nasal posterior. Punto más posterior en el plano sagital en el paladar duro } \\
\text { óseo. }\end{array}$ \\
\hline $\mathrm{H}$ & Parte superior de la lengua. Punto más superior de la lengua en relación a $\vee$ y $T$. \\
\hline $\mathrm{V}$ & Vallécula. Intersección de la epiglotis y la base de la lengua. \\
\hline GE & $\begin{array}{l}\text { Tubérculo Geni. Punto más posterior de la sínfisis mandibular y la parte antero-infe- } \\
\text { rior de la lengua }\end{array}$ \\
\hline NL. & Línea nasal. Línea entre la espina nasal anterior y la espina nasal posterior. \\
\hline UPW & $\begin{array}{l}\text { Pared faríngea superior. Punto de intersección de la línea perpendicular a la pared } \\
\text { posterior de la faringe desde la espina nasal posterior. }\end{array}$ \\
\hline MPW & $\begin{array}{l}\text { Pared faríngea media. Intersección de la línea perpendicular de } U \text { en la pared poste- } \\
\text { rior de la faringe }\end{array}$ \\
\hline LPW & $\begin{array}{l}\text { Pared faríngea inferior. Intersección de la línea perpendicular de } \vee \text { a la pared poste- } \\
\text { rior de la faringe. }\end{array}$ \\
\hline $\mathrm{AH}$ & Hioides anterior. Punto más anterior y superior en el cuerpo del hioides. \\
\hline CV & $\begin{array}{l}\text { Vértebras cervicales. Línea que recorre la parte anterior de la superficie de la 2a y } 3 a \\
\text { vértebra cervical. }\end{array}$ \\
\hline
\end{tabular}

y modificaciones introducidas por Samman et al, ${ }^{32}$. Para estos últimos autores, la cefalometría a pesar de ser un estudio en dos dimensiones, ha demostrado ser muy fiable para la medición del espacio aéreo posterior y se correlaciona altamente con la Tomografía Computarizada (CT Scan), con una precisión del $92 \%$, ellos, al igual que otros autores, sustentan que la posición inferior del hueso hiodes está relacionada con problemas respiratorios.

Para realizar el cefalograma se utilizan 17 variables, 12 medidas lineales, un ángulo, 4 medidas de área para la determinación de la permeabilidad de la nasofaringe, orofaringe y la hipofaringe. La lengua se mide en el plano sagital, su longitud (VT) y altura (H_lVT), la longitud del paladar blando (PM-U) y el espesor (SPT). El ancho del espacio de la vía aérea superior en el plano sagital (SPA), se describe en cuatro niveles: la nasofaringe (PM-UPW), la orofaringe (U-MPW), la hipofaringe (V-LPW) y la región de paso mínimo en las vías aéreas (PASmin). La posición del paladar blando está representada por el án- gulo (NL/PM-U) entre su eje mayor (PMU) y la línea nasal (NL). Además el área oral (OA) y área naso-orofaríngea (NOPA). (Fig. 4) $)^{30}$

A continuación se presentan los puntos, medidas y ángulos utilizados por los investigadores antes mencionados; siendo estos valores un marco de referencia para la población de Hong Kong ${ }^{32}$

Con referencia a este trazado, destaca la importancia de la evaluación de la posición de la lengua, hueso hioides y vértebras cervicales; es decir, considera los elementos que conforman la región cráneo-cérvico-mandibular con relación a las vías aéreas superiores. Estudia la vía aérea faríngea en sus tres niveles: nasofaringe, orofaringe e hipofaringe. Además puede determinarse la mínima distancia entre la base de la lengua y la pared posterior de la faringe, conocida como PASmin. Su utilidad es que provee información de las dimensiones anteroposteriores de los tejidos blandos, las cuales tienen características particulares diferenciales entre individuos sanos y pacientes con patologías 
en las vías aéreas superiores.

\section{I SCUS IÓN}

Para la evaluación de las vías aéreas se han empleado diversos trazados cefalométricos. Cada sección de la VAS reviste importancia clínica para el Odontólogo y por ende es motivo de estudio. Para algunos clínicos la sección más pequeña de la VAS tiene gran relevancia clínica porque infiere la dificultad del paso o conducción de los gases respiratorios.

Para McNamara la faringe inferior es la distancia mínima entre el punto donde el contorno posterior de la lengua cruzando el borde de la mandíbula y el punto más cercano en la pared posterior faríngea. Otros autores utilizan otro parámetro y la describen como la verdadera mínima dimensión del espacio aéreo. Dependiendo de la anatomía existente, aunque esta distancia puede no corresponder al diámetro verdaderamente menor del espacio de la vía aérea, se ha correlacionado positivamente con el volumen más pequeño medido detrás de la base de la lengua ${ }^{33}$.

Yueniwati et al. señalan que las radiografías laterales de cráneo son herramientas de diagnóstico valiosas y confiables para la detección del agrandamiento adenoideo con o sin obstrucción de las vías respiratorias. En su estudio, basado en el cefalograma de McNamara, muestra una sensibilidad de 68,4\%, especificidad 100\%, valor predictivo positivo $100 \%$, valor predictivo negativo $45,4 \%$ y precisión $75 \%$. Concluyendo que usando el método de McNamara es posible predecir la obstrucción de las vías respiratorias $^{34}$. En concordancia a lo expresado por Quiroga et al., la radiografía cefálica lateral se considera una importante herramienta de detección confiable para determinar la necesidad de una evaluación otorrinolaringológica en pacientes con hipertrofia adenoidea ${ }^{35}$.

En relación al espacio faríngeo, conocer el diámetro superior e inferior del mismo, su relación y efecto sobre el crecimiento facial es un tema aún por resolver y algunos investigadores consideran, que un cefalograma no es una herramienta diagnóstica suficiente a considerar en estos casos, en los cuales se requieren otros estudios más específicos. Al respecto, se ha discutido que es difícil establecer si la obstrucción de las vías respiratorias superiores, es responsable de la inducción de cambios en la morfología craneofacial, o si estos cambios son genéticamente determinados, facilitando la obstrucción por pequeños aumentos del volumen adenoi-
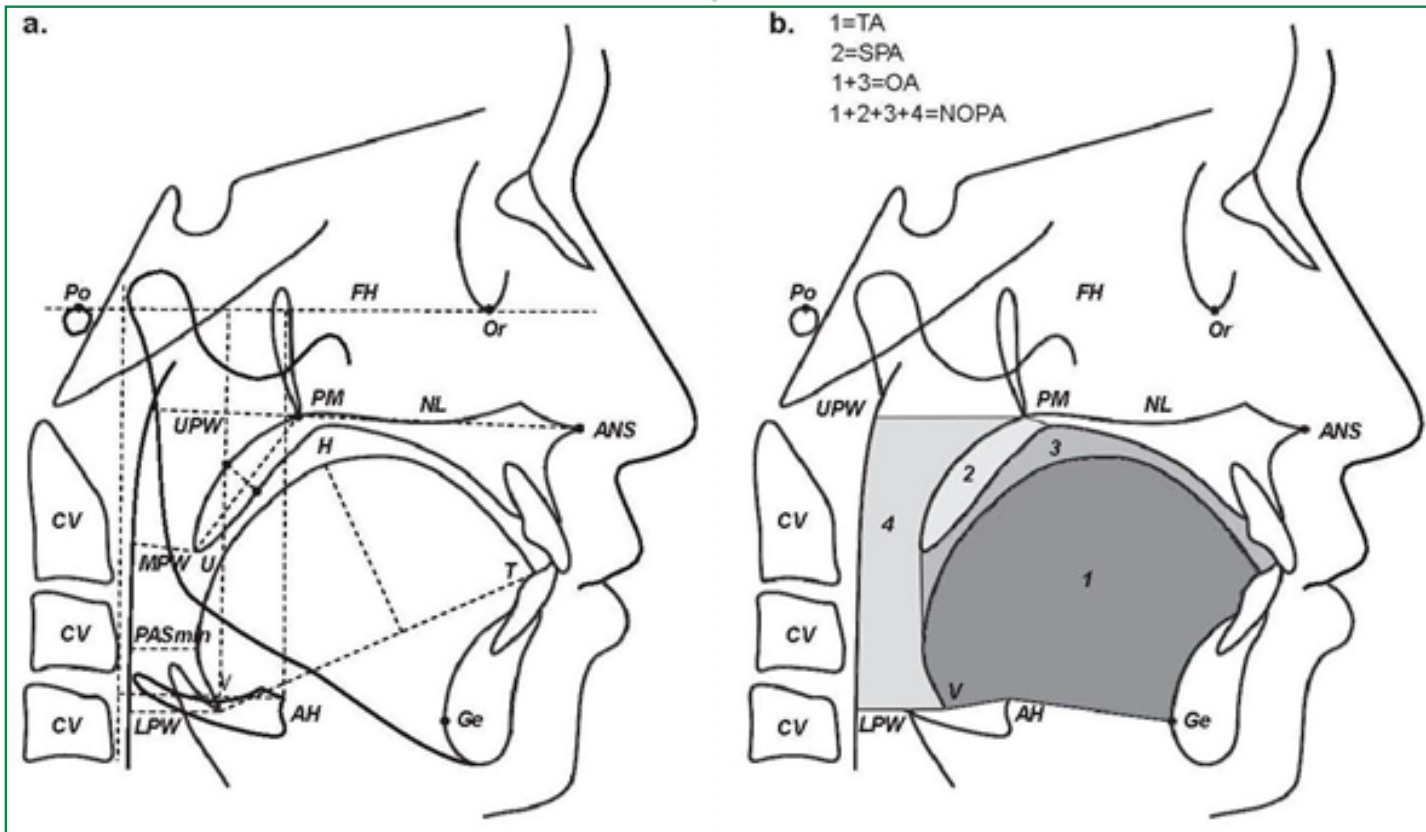

Figura 4. Cefalograma de Lyberg, Krogstad, Djupesland con modificaciones de Samman, Mohammadi y Xia.

QUEVEDO-PIÑA M HERNÁNDEZ-

ANDARA A

ZAMBRANO E

VANDA D

EVALUACIÓN DE

LAS VÍAS AÉREAS

SUPERIORES

A TRAVÉS DE

TRAZADOS

CEFALOMÉTRICOS

283
REV, ODONTOL.

UNIV. CID. SÃo PAULO

2017; 29(3): 276-88, SET-DEZ 


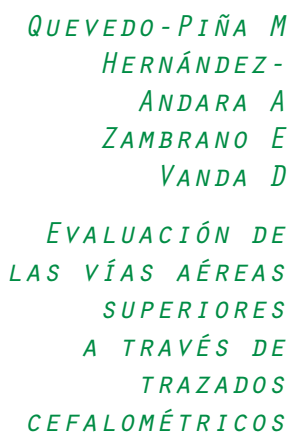

REV, ODONTOL UNIV. CID, SÃO PAULO

Cuadro 3. Definiciones de áreas en $\mathrm{mm}^{2}$ y proporciones.

\begin{tabular}{|c|c|}
\hline $\mathrm{AH}-\mathrm{CV}$ & Posición del hueso hioides en el plano horizontal, de $\mathrm{AH}$ a CV y paralela a FH \\
\hline $\mathrm{AH} \_\mathrm{FH}$. & Posición del hueso hioides en el plano vertical, perpendicular de $\mathrm{AH}$ a $\mathrm{FH}$ \\
\hline $\mathrm{H} \perp$ VT. & $\begin{array}{l}\text { Altura de la lengua, medida como la distancia perpendicular de } \mathrm{H} \text { a la línea } \\
\text { VT }\end{array}$ \\
\hline NL / PM-U. & Inclinación del eje longitudinal del paladar blando, relativo a la línea nasal. \\
\hline PASmin. & La distancia más corta entre la base de la lengua y la pared posterior faríngea. \\
\hline PM-U. & Longitud del paladar blando, distancia desde PM a U. \\
\hline PM-UPW. & Ancho de la vía aérea nasofaríngea de PM a UPW \\
\hline SPT. & $\begin{array}{l}\text { Espesor de paladar blando, representa el máxima grosor del paladar blando } \\
\text { medido perpendicularmente a la línea PM-U. }\end{array}$ \\
\hline U-MPW & Ancho de la vía aérea orofaríngea. Distancia desde U a MPW \\
\hline $\mathrm{V}-\mathrm{CV}$ & $\begin{array}{l}\text { Posición de la vallécula en el plano horizontal, línea desde } \vee \text { a CV y paralela } \\
\text { a FH }\end{array}$ \\
\hline $\mathrm{V} \perp \mathrm{FH}$ & Posición de la vallécula en el plano vertical, línea perpendicular de $\mathrm{V}$ a FH. \\
\hline V-LPW & Ancho de la vía aérea hipofaríngea desde $V$ a LPW. \\
\hline VT & Longitud de la lengua, medida como la distancia desde $\mathrm{V}$ a $\mathrm{T}$ \\
\hline $\mathrm{AH}-\mathrm{CV}$ & Posición del hueso hioides en el plano horizontal, de $\mathrm{AH}$ a CV y paralela a FH \\
\hline $\mathrm{AH} \perp \mathrm{FH}$ & Posición del hueso hioides en el plano vertical, perpendicular de $\mathrm{AH}$ a FH \\
\hline $\mathrm{H} \perp \mathrm{VT}$ & $\begin{array}{l}\text { Altura de la lengua, medida como la distancia perpendicular de } \mathrm{H} \text { a la línea } \\
\text { VT }\end{array}$ \\
\hline NL / PM-U. & Inclinación del eje longitudinal del paladar blando, relativo a la línea nasal. \\
\hline
\end{tabular}

\section{Cuadro 4 ou continuação do 3 ?}

Zona oral, Área oral calculada que incluye el área de la lengua y que se extiende supeOA riormente al contorno del paladar blando y duro

Área naso- Área trazada calculada incluyendo OA y el área definida por los puntos de orofaríngea, PM, UPW, LPW y $\vee$ a lo largo de la pared posterior de la faringe y el contorno NOPA dorsal de la lengua incluyendo la zona del paladar blando (SPA).

Zona SPA Corresponde al contorno antero-posterior del paladar blando, el límite superior paladar es una línea perpendicular a la PM y a la línea PM-U.

blando.

SPA / Relación entre el paladar blando y la diferencia entre el área naso-orofaríngea

(NOPA-OA) y las áreas orales, corresponde al área faríngea.

Área Len- El trazado del contorno dorsal de la lengua de desde $\mathrm{V}$ a través de $\mathrm{H}$ a T. El

gua TA. contorno inferior se redujo a un polígono geométrico cuyo límite está definido por los segmentos de línea que conectan los puntos $\mathrm{V}, \mathrm{AH}, \mathrm{GE}, \mathrm{y} \mathrm{T}$.

TA/OA. Relación lengua y cavidad bucal

TA+SPA/ Relación área de lengua mas paladar blando y área naso orofaríngea. TA+SPA/ NOPA NOPA

SPA/NOPA- Relación entre área de paladar blando y la diferencia entre área naso orofarín-OA gea y área oral, es decir área faríngea.

deo. ${ }^{27,36}$

Sin embargo una perspectiva diferente ha sido planteada por otros autores, quienes han considerado que alteraciones como la respiración bucal, conllevan influencias perjudiciales en el proceso diná- mico de crecimiento y desarrollo facial; y una de las causas en la modificación del proceso respiratorio, puede estar dada por la hipertrofia de las amígdalas faríngeas y palatina, que obstruyen los espacios nasales y bucofaríngeo. Aquellos cambios 
inducidos en el paciente, no son más que ajustes compensatorios neuromusculares para el nuevo patrón de respiración no fisiológica y estos pueden ser medidos. ${ }^{25}$

Es importante acotar, que la fiabilidad de los métodos para evaluación de vías aéreas superiores ha sido estudiada en los últimos años. Para Bianchi et al. ${ }^{37}$ (2016), la tomografía computarizada ofrece ventajas significativas cuando es comparada con la radiografía, debido a que permite una mejor delimitación de los tejidos y del espacio aéreo; siendo más precisa en la medición y la observación de la morfología de estas estructuras. En su investigación analiza las vías respiratorias en 3D donde observo un incremento significativo del volumen de la vía aérea total con un ensanchamiento más prominente en sentido medio-lateral que antero posterior $^{37}$.

A pesar de los métodos imagenológicos usados en esta área (TAC y RM) la radiografía cefálica lateral con su respectivo análisis siguen siendo una herramienta útil para la evaluación y diagnóstico de problemas en las VAS. En tal sentido, Quiroga et al. utilizaron diferentes métodos para medir hipertrofia adenoidea en niños respiradores bucales, entre los cuales estaban la medición lineal de McNamara y el análisis trapezoidal de Handelmann y Osborne. En esa investigación, los valores más confiables y homogéneos fueron las medidas lineales de McNamara. El análisis trapezoidal tuvo valores mayores de especificidad $94 \%$, sensibilidad $75 \%$, valor predictivo positivo $95 \%$ y valor predictivo negativo $72 \%$. Estos resultados confirman la validez de la cefalometría para evaluar la hipertrofia adenoidea en niños de 6 a 12 años. De manera opuesta a lo expresado, otros autores recomiendan la utilización del análisis trapezoidal, ya que en su opinión este método refleja los límites de la nasofaringe de una manera más precisa $^{38}$.

Con referencia al cefalograma de Lyberg, Krogstad y Djupesland con modificaciones de Samman, Mohammadi y Xia, se puede decir que permite obtener medidas de la vía aérea superior, utilizando tamaño de la lengua, paladar blando, nasofaringe, orofaringe, hipofaringe, al igual que la posición relativa del hueso hioides y vallécula. Guttal y Burde estudiaron la población India obteniendo correlaciones positivas con los sujetos de nacionalidad china, logrando generar aportes para futuros estudios y poder evaluar las dimensiones de las vías respiratorias en diferentes maloclusiones esqueléticas, planteando además otra fortaleza como es la utilización del trazado cefalómetrico para la planificación de casos quirúrgicos y en post tratamiento. De hecho sus resultados han permitido que sean tomados como patrones de referencia para futuras investigaciones ${ }^{39}$.

\section{CONCLUSIÓN}

La cefalometría se considera una herramienta diagnóstica de gran importancia, dado que permite conocer las relaciones anatómicas entre las bases óseas y los tejidos blandos. Adquiere especial relevancia para el clínico, al medir los cambios evolutivos provocados a consecuencia de la aplicación del tratamiento y es una guía que le permite reorientar, de ser necesario, sus objetivos terapéuticos. La vista lateral del cráneo favorece la medición, análisis, ubicación, determinación de la configuración y el crecimiento del tejido adenoideo.

Esta revisión destaca diferentes análisis cefalométricos, que pueden ser utilizados para evaluar las vías aéreas superiores, permitiendo conocer la situación inicial y los cambios posteriores a la aplicación de un factor modificador, ya sea ortodóncico, ortopédico, maxilar y/o quirúrgico. Entre los diferentes trazados presentados, el equipo de salud deberá dilucidar cuál es el cefalograma adecuado a los requerimientos diagnósticos y terapéuticos de cada paciente y de la especialidad del profesional tratante.

El trazado cefalométrico de Lyberg, Krogstad y Djupesland, con modificaciones de Samman, Mohammadi y Xia, además de evaluar las vías aéreas superiores incorpora la región cervical y la estructura hioidea que recibe gran influencia de la lengua, considerando de esta manera la unidad cráneo-cérvico- mandibular, por lo que sugerimos el uso de este trazado.
QUEVEDO-PIÑA M HERNÁNDEZ-

ANDARA A

ZAMBRANO E

VANDA D

EVALUACIón DE

LAS VÍAS AÉREAS

SUPERIORES

a tRAVÉS DE

TRAZADOS

CEFALOMÉTRICOS

\section{5}

REV, ODONTOL.

UNIV. CID, SÃo

PAULO

2017; 29(3): $276-88$, SET - DEZ 
QUEVEDO-PIÑA M

HERNÁNDEZ-

ANDARA A

ZAMBRANO E

VANDA D

EVALUACIÓN DE LAS VIIAS AÉREAS

SUPERIORES

A TRAVÉS DE

TRAZADOS

CEFALOMÉTRICOS
REV, ODONTOL.

UNIV, CID, SÃO PAULO:

2017; 29(3): $276-88$, SET - DEZ

\section{REFERÊNCIAS}

1. Graber T, Vanarsdall R, Vig JR K. Ortodoncia: principios y técnicas actuales. 4 ed. España: Elsevier; 2006.

2. Juliano ML, Machado MA, de CarvaIho LB, Zancanella E, Santos GM, do Prado LB, et al. Polysomnographic findings are associated with cephalometric measurements in mouth-breathing children. Journal of clinical sleep medicine : JCSM : official publication of the American Academy of Sleep Medicine. 2009;5(6):554-61.

3. Villafranca FdC, Cobo Plana J, Fernández Mondragón MP, Jiménez A. Cefalometría de las vías aéreas superiores (VAS). RCOE. 2002;7:407-14.

4. Consenso Nacional sobre el síndrome de apneas-Hipopneas del sueño (SAHS) - Resumen. Arch Bronconeumol. 2005;41(Supl 4):7-9.

5. Vargas Aragonés C. Horizontal verdadera: una línea de referencia más relevante para el análisis cefalométrico. Odovtos. 2004(6):20-4.

6. Merí A. Fundamentos de fisiología de la actividad física y el deporte. Buenos Aires: Panamericana; 2005.

7. Welsch U, Sobotta J. Histología. España: Panamericana; 2010.

8. Varela MAR, Pastor AC. Síndrome del respirador bucal: aproximación teórica y observación experimental: M.A. Ruiz; 2001.

9. Han S, Choi YJ, Chung CJ, Kim JY, Kim $\mathrm{KH}$. Long-term pharyngeal airway changes after bionator treatment in adolescents with skeletal Class II malocclusions. Korean journal of orthodontics. 2014;44(1):13-9.

10. Laxmi NV, Talla H, Meesala D, Soujanya S, Naomi N, Poosa M. Importance of cephalographs in diagnosis of patients with sleep apnea. Contemporary clinical dentistry. 2015;6(Suppl 1):S221-6.
11. Carulla Martínez D, Espinosa Quiros D, Mesa Levy T. Estudio cefalométrico del hueso hioides en niños respiradores bucales de 11 años.: Segunda parte. Revista Cubana de Estomatología. 2010;47(2):178-88.

12. Valiathan M, El H, Hans MG, Palomo MJ. Effects of extraction versus non-extraction treatment on oropharyngeal airway volume. The Angle orthodontist. 2010;80(6):1068-74.

13. Brons S, van Beusichem ME, Bronkhorst EM, Draaisma J, Berge SJ, Maal TJ, et al. Methods to quantify soft-tissue based facial growth and treatment outcomes in children: a systematic review. PloS one. 2012;7(8):e41898.

14. Puigdollers Pérez A. Más investigación y conocimiento sobre la tomografía computarizada de haz cónico Rev Esp Ortod 2011;41(1):03-4.

15. Zamora Martinez N. Evaluación de las dosis de radiación con los sistemas de tomografía computarizada de haz cónico en ortodoncia. Rev Esp Ortod. 2011;41(1):17-22.

16. Kurt G, Sisman C, Akin E, Akcam T. Cephalometric comparison of pharyngeal airway in snoring and non-snoring patients. European journal of dentistry. 2011;5(1):84-8.

17. Grewal N, Godhane AV. Lateral cephalometry: A simple and economical clinical guide for assessment of nasopharyngeal free airway space in mouth breathers. Contemporary clinical dentistry. 2010;1(2):66-9.

18. Zhong Z, Tang Z, Gao X, Zeng XL. A comparison study of upper airway among different skeletal craniofacial patterns in nonsnoring Chinese children. The Angle orthodontist. 2010;80(2):267-74.

19. Solow B. The pattern of craniofacial associations : A morphological and methodological correlation and factor analysis study on young male adults : With an appendix by Søren Johansen. Cph.: (tr. : Aarhuus Stiftsbogtrykkerie); 1966. 
20. Villanueva $P$, Valenzuela S, Santander $H$, Zúñiga $C$, Ravera $M$, Miralles $R$. Efecto de la postura de cabeza en mediciones de la vía aérea. Revista CEFAC. 2004;6(1):44-8.

21. Cárdenas JM, Flores Flores JC, Gutiérrez Cantú FJ, Cárdenas GM, Sánchez Meraz W, Guerrero Barrera AL. Estudio Morfométrico de la Posición Cráneo-Cervical en Pacientes con Clases Esqueletales II y III. International Journal of Morphology. 2015;33(2):415-9.

22. Solow B, Kreiborg S. Soft-tissue stretching: a possible control factor in craniofacial morphogenesis. Scandinavian journal of dental research. 1977;85(6):505-7.

23. McNamara JA. A method of cephalometric evaluation. American journal of orthodontics. 1984;86(6):449-69.

24. Gouveia SAdS, Nahás ACR, Cotrim-Ferreira FA. Estudo cefalométrico das alterações dos terços médio e inferior da face em pacientes com diferentes padrões respiratórios e faciais. Revista Dental Press de Ortodontia e Ortopedia Facial. 2009;14(4):92-100.

25. Alcazar NMPV, Freitas MRd, Janson G, Henriques JFC, Freitas KMSd. Estudo cefalométrico comparativo dos espaços naso e bucofaríngeo nas más oclusões Classe I e Classe II, Divisão 1, sem tratamento ortodôntico, com diferentes padrões de crescimento. Revista Dental Press de Ortodontia e Ortopedia Facial. 2004;9(4):68-76.

26. Mayoral J, Mayoral G, Mayoral P. Ortodoncia principios fundamentales $y$ práctica. 6 ed. Barcelona: Editorial Labor; 1990.

27. Barbosa MdCe, Knop LAH, Lessa MM, Araujo TMd. Avaliação da radiografia cefalométrica lateral como meio de diagnóstico da hipertrofia de adenoide. Revista Dental Press de Ortodontia e Ortopedia Facial. 2009;14(4):83-91.

28. Handelman CS, Osborne G. Growth of the nasopharynx and adenoid development from one to eighteeen years. The Angle orthodontist. 1976;46(3):243-59.
I SSN 1983-5183

29. Fujioka M, Young LW, Girdany BR. Radiographic evaluation of adenoidal size in children: adenoidal-nasopharyngeal ratio. AJR American journal of roentgenology. 1979;133(3):401-4.

30. Tangugsorn $\mathrm{V}$, Skatvedt O, Krogstad O, Lyberg T. Obstructive sleep apnoea: a cephalometric study. Part II. Uvulo-glossopharyngeal morphology. European journal of orthodontics. 1995;17(1):57-67.

31. Linder-Aronson S, Woodside DG, Daigle DJ. A longitudinal study of the growth in length of the maxilla in boys between ages 6-20 years. Transactions European Orthodontic Society. 1975:169-79.

32. Samman N, Mohammadi H, Xia J. Cephalometric norms for the upper airway in a healthy Hong Kong Chinese population. Hong Kong medical journal = Xianggang yi xue za zhi. 2003;9(1):25-30.

33. Daraze A, Delatte M, Liistro G, Majzoub Z. Cephalometrics of Pharyngeal Airway Space in Lebanese Adults. International journal of dentistry. 2017;2017:3959456.

34. Yueniwati Y, Halim N. Diagnostic Test Value of Assessment Adenoid Enlargement with and Without Airway Obstruction Using Lateral Soft Tissues X-ray Compared to Nasoendoscopy. Indian Journal of Otolaryngology and Head \& Neck Surgery.1-6.

35. Souki MQ, Souki BQ, Franco LP, Becker HM, Araujo EA. Reliability of subjective, linear, ratio and area cephalometric measurements in assessing adenoid hypertrophy among different age groups. The Angle orthodontist. 2012;82(6):1001-7.

36. Ronchi P, Cinquini V, Ambrosoli A, Caprioglio A. Maxillomandibular advancement in obstructive sleep apnea syndrome patients: a restrospective study on the sagittal cephalometric variables. Journal of oral \& maxillofacial research. 2013;4(2):e5.
QUEVEDO-PIÑA M HERNÁNDEZ-

ANDARA A

ZAMBRANO E

VANDA D

EVALUACIÓN DE

LAS VÍAS AÉREAS

SUPERIORES

A TRAVÉS DE

TRAZADOS

CEFALOMÉTRICOS

287

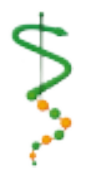

REV, ODONTOL.

UNIV, CID, SÃO

PAULO

2017; 29(3):

$276-88$, SET - DEZ 
QUEVEDO-PIÑA M HERNÁNDEZANDARA A

ZAMBRANO E

VANDA D

EVALUACIÓN DE LAS VÍAS AÉREAS SUPERIORES

A TRAVÉS DE TRAZADOS CEFALOMÉTRICOS
37. Bianchi FA, Gerbino G, Corsico M, Schellino E, Barla N, Verze $L$, et al. Soft, hard-tissues and pharyngeal airway volume changes following maxillomandibular transverse osteodistraction: Computed tomography and three-dimensional laser scanner evaluation. Journal of cranio-maxillo-facial surgery : official publication of the European Association for Cranio-Maxillo-Facial Surgery. 2017;45(1):4755.
38. Ansar J, Maheshwari S, Verma SK, Singh RK, Agarwal DK, Bhattacharya P. Soft tissue airway dimensions and craniocervical posture in subjects with different growth patterns. The Angle orthodontist. 2015;85(4):604-10.

39. Guttal KS, Burde KN. Cephalometric evaluation of upper airway in healthy adult population: a preliminary study. Journal of Oral and Maxillofacial Radiology. 2013;1(2):55.

Recebido em 14/08/2017

Aceito em 14/08/2017 